\title{
TAGUNG
}

\section{Internationale Kooperation im Bereich erneuerbare Energien: Ein Blick auf die Schwellenländer}

\author{
Sybille Röhrkasten*
}

Seit der Energiewende 2011 prägt der Ausbau erneuerbarer Energien das politische Tagesgeschäft in Deutschland. Deutsche Energiepolitik gleicht häufig einer Nabelschau. Außerhalb eines engen Entscheidungsträger- und Expertenkreises wird die internationale Dimension der Energiepolitik ausgeblendet. Gleichzeitig schaut die Welt mit einer Mischung aus Verwunderung und Anerkennung auf Deutschland. Der Erfolg der deutschen Energiewende wird stark beeinflussen, ob andere Staaten Energietransformationen als realisierbar einschätzen und ihre Politik entsprechend umsteuern. Auf dem Gebiet der erneuerbaren Energien gilt Deutschland schon lange als internationaler Vorreiter. Die Einspeisevergütung für erneuerbare Energien, die Deutschland bereits im Jahr 2000 einführte, ist ein Exportschlager. In seiner Entwicklungspolitik setzt sich Deutschland seit Langem für erneuerbare Energien ein. So ist die Gründung der ,International Renewable Energy Agency“ (IRENA) im Jahr 2011 ein Erfolg deutscher Diplomatie.

Vor diesem Hintergrund befasste sich die Konferenz mit zwei zentralen Herausforderungen, vor denen die internationale Energiepolitik heute steht: der Notwendigkeit eines umfassenden Ausbaus erneuerbarer Energien und dem Aufstieg der Schwellenländer, die auch in der Energiepolitik das internationale Machtgefüge entscheidend verändern. Die Konferenz fand am 21. Juni 2012 in der Stiftung Wissenschaft und Politik (SWP) im Rahmen des internationalen Forschungsprojekts „Challenges of European External Energy Governance

\section{International Governance for Renewable Energy and the Role of Emerging Powers}

Eine Tagung der Stiftung Wissenschaft und Politik (SWP) und des Arbeitskreises Europäische Integration e.V. (AEI) im Rahmen des Forschungsprojekts „Challenges of European External Energy Governance with Emerging Powers“

Mit freundlicher Unterstützung der Europäischen Kommission, der VolkswagenStiftung, der Compagnia di San Paolo und des Riksbankens Jubileumsfond

Berlin, 21. Juni 2012

\section{Introduction}

Kirsten WESTPHAL, Stiftung Wissenschaft und Politik, Berlin

$A$ vision for a global energy transition

Carsten HOYER-KLICK, Deutsches Zentrum für Luft und Raumfahrt, Köln

Emerging Powers' Renewable Energy

Governance

Chair

Michèle KNODT, Technische Universität Darmstadt

China

ZHA Daojiong, Peking University, Beijing

India

Deepti MAHAJAN, The Energy and Resources Institute, New-Delhi

Brazil

Roberto SCHAEFFER, Federal University of Rio de Janeiro, Rio de Janeiro

* Dipl.-Vw. Sybille Röhrkasten, M.A., Doktorandin in der Forschungsgruppe Globale Fragen, Stiftung Wissenschaft und Politik, Berlin. 
with Emerging Powers: Meeting Tiger, Dragon, Lion and Jaguar" statt. Das von der Technischen Universität Darmstadt koordinierte Forschungsprojekt verbindet Universitäten und Forschungsinstitute aus Brasilien, China, Indien, Südafrika und verschiedenen europäischen Staaten.

In der Begrüßung verwies Kirsten Westphal auf zwei einjährige Jubiläen, die für die Konferenz von Bedeutung seien: den ersten Jahrestag der deutschen Energiewende und das einjährige Bestehen der IRENA. Sie stellte die zentralen Komponenten des deutschen Energiekonzepts vor, das neben dem Ausbau erneuerbarer Energien und dem Ausstieg aus der Atomenergie auch die Bereiche Energieeinsparung und Energieeffizienz umfasse. Sie unterstrich, dass sich das Energiekonzept allein auf die interne Dimension der Energiepolitik bezieht. Bezüglich der IRENA verwies sie auf die fragmentierte und veraltete institutionelle Landschaft internationaler Energiepolitik und betonte, ihre zentrale Aufgabe müsse sein, die Kohärenz internationaler Kooperation mit Blick auf erneuerbare Energien zu stärken.

\section{Die globale Energiewende als internationale Herausforderung}

Da gerade in der Energiepolitik ein Austausch zwischen Sozial- und Naturwissenschaft von zentraler Bedeutung ist, begann der Tag mit einem naturwissenschaftlichen Vortrag von Carsten Hoyer-Klick zur Vision einer globalen Energietransition. Er zeigte zentrale Bestandteile einer Energietransition auf und leitete daraus Aufgaben für die internationale Politik ab. Nach Angaben von Hoyer-Klick steht das globale Energiesystem vor zwei zentralen Herausforderungen: der Notwendigkeit, die globale Erwärmung auf zwei Grad Celsius zu begrenzen und gleichzeitig die stark ansteigende globale Energienachfrage zu befriedigen. Ohne umfassende Investitionen in erneuerbare Energien sei dies nicht zu meistern.

Groß angelegte Investitionen in erneuerbare Energien unterblieben häufig aufgrund hoher Anfangskosten, die sich durch Energieeinspa-
South Africa

Judy SMITH-HÖHN, South African Institute of International Affairs, Pretoria

International Governance for Renewable Energy

Chair

Yonatan SCHVARTZMAN, Aarhus University, Aarhus

An introduction to IRENA

Francisco BOSHELL, International Renewable Energy Agency, Bonn

Institutional landscape for renewable energy promotion

Sybille RÖHRKASTEN, Stiftung Wissenschaft und Politik, Berlin

Comments: IRENA, the institutional landscape and a plea for IFETA (International Fossil Energy Termination Agency)

Gerhard KNIES, DESERTEC Foundation / DESERTEC University Network (DUN), Hamburg

Engaging Emerging Powers in International Governance

Chair

Jens STEFFEK, Technische Universität Darmstadt

EU external energy dialogue with emerging powers Marion WILDE, Directorate General Energy, European Commission, Brussels

Engaging emerging powers in international cooperation for renewable energy

Thijs VAN DE GRAF, Ghent Institute of International Studies, Ghent

rungen und Kostensenkungen erst langfristig auszahlten. Eine umfassende Marktintegration erneuerbarer Energien erfordere weitreichende Veränderungen bestehender Marktstrukturen. Märkte müssten an die Schwankungen des Angebots erneuerbarer Energien angepasst werden. Neue Lösungen im Bereich der Energiespeicherung seien notwendig. Zudem müssten neue Preisstrukturen gefunden werden, welche sich nicht mehr an Grenzkosten orientieren, da erneuerbare Energien über sehr geringe beziehungsweise keine Grenzkosten verfügten. Auch über die Bildung von Kapazitätsmärkten 
sei nachzudenken. Zudem sei zu bedenken, dass die langfristig zu erwartenden Preissenkungen aufgrund der Marktintegration erneuerbarer Energien geringe Anreize für Investitionen im Elektrizitätssektor böten.

Der politische und wirtschaftliche Rahmen sei für einen umfassenden Ausbau erneuerbarer Energien von zentraler Bedeutung. Für eine angemessene Rahmensetzung fehle häufig das notwendige Wissen. So seien umfangreiche technologische und sozioökonomische Daten zur Bestimmung des technischen und ökonomischen Potenzials von erneuerbaren Energien und der Ermittlung von Energieszenarien erforderlich. Der Austausch über, best and worst practices' sei für die Entwicklung angemessener politischer und finanzieller Anreizinstrumente zentral. Wichtigste Aufgaben für internationale Kooperation seien daher Wissensaustausch und gegenseitiges Lernen.

\section{Die Förderung erneuerbarer Energien in China, Indien, Brasilien und Südafrika}

Die folgenden vier Vorträge richteten den Blick auf die Governance erneuerbarer Energien in China, Indien, Brasilien und Südafrika. Dabei ging es zunächst um die nationalen Kontexte und nationale Politiken zur Förderung erneuerbarer Energien. Die ReferentInnen zeigten auf, inwiefern die vier Staaten in die internationale Kooperation eingebunden sind und welche Präferenzen sie in der internationalen Politik verfolgen.

Zha Daojiong verwies zunächst auf die unzureichende Datenlage in China, die eine umfassende Darstellung des nationalen Kontextes erneuerbarer Energien erschwere. In China sei die Förderung ländlicher Entwicklung zentrale Triebkraft für den Ausbau erneuerbarer Energien gewesen. 1985 wurde ein Programm verabschiedet, um die Abhängigkeit von Feuerholz zu reduzieren. Nach einer Flutkatastrophe im Jahr 1998 sah man in der Förderung erneuerbarer Energien die Möglichkeit, in den betroffenen Regionen ökonomische Alternativen zu entwickeln. Zentrale Dokumente für die na- tionale Politik mit Bezug zu Erneuerbaren Energien seien die Weißbücher zur Energiepolitik (2007) und zum Klimaschutz (2011). Zha gab zu bedenken, dass erneuerbare Energien in China trotz erzielter Fortschritte nach wie vor mit einer Reihe von Hindernissen konfrontiert seien: die Kohleproduktion gehe mit negativen Begleiterscheinungen einher, wie zum Beispiel Umweltverschmutzung, deren Kosten oft nicht vollständig berücksichtigt würden, der Fokus müsse stärker auf Investitionen in Erzeugungskapazitäten anstatt in die Stromproduktion gelegt werden und die Ziele zum Ausbau erneuerbarer Energien seien nicht verpflichtend. Zudem beeinträchtige mangelnder Wettbewerb die Innovationsfähigkeit in China.

Erneuerbare Energien seien eine der vier zentralen Säulen chinesischer Energiediplomatie. Die drei weiteren Säulen seien die Sicherung des Zugangs zu fossilen Energieträgern, finanzielle Aspekte des internationalen Energiehandels sowie Klimaschutz. In China herrsche eine Unzufriedenheit mit dem Agenda-Setting und dem Framing von Themen in der internationalen Politik. So sei China in der internationalen Klimapolitik gegen eine Orientierung an absoluten Emissionen, sondern befürworte ProKopf-Emissionen als Maßzahl. China habe ein großes Interesse am Transfer von Know-how, finanziellen und technischen Ressourcen. Grundsätzlich befürworte China Verhandlungen im Rahmen der Vereinten Nationen, während bei minilateralen Foren versteckte Agenden befürchtet würden. Beim Betrieb von Produktionsanlagen akzeptiere China internationale Standards. Gleichzeitig strebe es an, dass eigene technologische Innovationen international als Standards akzeptiert würden. Insgesamt erachtete Zha es als wenig wahrscheinlich, dass China in Zukunft Initiativen zur grenzüberschreitenden Förderung erneuerbarer Energien ergreifen werde.

Deepti Mahajan unterstrich, dass 20 Prozent der Stromerzeugungskapazität in Indien auf Großwasserkraft und weitere 12 Prozent auf anderen Formen erneuerbarer Energien basiere. Nach wie vor hinge Indiens Energieversor- 
gung jedoch stark von Kohlekraft ab. Unter den netzintegrierten erneuerbaren Energien sei die Windkraft am wichtigsten; Biomasse dominiere die netzferne Versorgung mit erneuerbaren Energien. Die indische Regierung fördere erneuerbare Energien seit den 1970er Jahren. Zentrale Prioritäten seien der Zugang zu Energie und die Befriedigung energiebezogener Grundbedürfnisse, dezentrale Ansätze und die Verringerung der Importabhängigkeit.

Mahajan betonte, dass Indien in verschiedene internationale Kooperationsansätze zur Förderung erneuerbarer Energien eingebunden sei. Die Aktivitäten der IRENA stießen in Indien auf großes Interesse. Dort sei das Südasien-Sekretariat der ,Renewable Energy and Energy Efficiency Partnership ' (REEEP) beheimatet und 2010 wurde die ,Delhi International Renewable Energy Conference‘ organisiert. Des Weiteren sei Indien aktives Mitglied des, Clean Energy Ministerial' (CEM) und habe ein großes Interesse an der Erfassung von Potenzialen zur Nutzung erneuerbarer Energien und der Bewertung von Marktpotenzialen und Politiklösungen. Außerdem sei es an Investitionen, Maßnahmen zur Forschung und Entwicklung und am Kapazitätsaufbau interessiert. Auch die Bereiche Technologietransfer und Klimafinanzierung seien für Indien von großer Bedeutung. Insgesamt unterstrich Mahajan die Bedeutung von Wissensnetzwerken für die internationale Kooperation. Andere Staaten könnten auch von den Erfahrungen Indiens profitieren. Genauso wie auf nationaler Ebene sehe Indien den Zugang zu Energie als ein wichtiges Thema für die internationale Politik. Abschließend betonte Mahajan, dass sowohl Industrie- als auch Entwicklungsländer sicher stellen müssten, dass die gegenwärtige Finanzkrise den Ausbau erneuerbarer Energien nicht abbremse.

Roberto Schaeffer unterstrich die Errungenschaften Brasiliens bei der Förderung erneuerbarer Energien. Der brasilianische Energiemix basiere zu 45 Prozent auf erneuerbaren Energien. Neben der Wasserkraft, die zu 80 Prozent zur Elektrizitätsgewinnung beitrage, seien ins- besondere Biokraftstoffe von Bedeutung. So würden in Brasilien nur noch Fahrzeuge mit Flex-Fuel-Motoren hergestellt. Autofahrer könnten an der Tankstelle unterschiedliche Biokraftstoffanteile wählen. Der Klimawandel errege in Brasilien große Besorgnis. Aufgrund der hohen Abhängigkeit von Wasserkraft wirke er sich direkt auf die Energieverfügbarkeit aus. Brasilien sei zunehmend am Ausbau von Wind- und Solarenergie interessiert. Windenergie sei in Brasilien mittlerweile wettbewerbsfähig. Die Verfügbarkeit von Windenergie wirke komplementär zur Wasserkraft. Aufgrund der Dominanz von Wasserkraft werde jedoch weder Wind- noch Solarenergie eine große Rolle spielen. Schaeffer betonte zudem, dass es in Brasilien keine Flächenknappheit gebe. Die Produktion von Biokraftstoffen stehe daher weder in Konkurrenz zur Nahrungsmittelproduktion noch trage sie direkt zur Entwaldung bei. Da 95 Prozent der Brasilianer Zugang zu Energie hätten, sei Elektrifizierung kein Thema mehr.

In seiner Außenpolitik setze Brasilien vor allem auf die Förderung seiner Ethanolexporte. Problematisch sei, dass Industrieländer sich zwar für den Freihandel aussprächen, gleichzeitig aber Importe aus Entwicklungsländern verhinderten. Aufgrund der eigenen Errungenschaften bei der Förderung erneuerbarer Energien brauche Brasilien keine Technologietransfers von außen. Eine Vertreterin der brasilianischen Botschaft in Berlin ergänzte, dass sich Brasilien international in der, Global Bioenergy Partnership“ (GBEP) engagiere. Der IRENA sei Brasilien nicht beigetreten, da Biomasse und Wasserkraft nicht ausreichend berücksichtigt würden. Zudem fördere Brasilien im Rahmen von Süd-Süd-Kooperation den Transfer von Biokraftstoff-Technologien.

Judy Smith-Höhn unterstrich zunächst, dass die Elektrizitätsversorgung in Südafrika zu 90 Prozent auf Kohle basiere. Die damit einhergehenden Treibhausgasemissionen böten zunehmend Anlass zur Sorge. Klimaschutz und das Bestreben, die Energieversorgung zu diversifizieren, weckten auch in Südafrika ein 
Interesse an erneuerbaren Energieträgern. So sprach sich das ,Weißbuch erneuerbare Energien` aus dem Jahr 2004 für eine verstärkte Nutzung erneuerbarer Energien aus. Der ,Integrated Resource Plan 2010-2030`, der sich gegenwärtig in Überarbeitung befinde, sehe für neu zu installierende Stromerzeugungskapazitäten einen Anteil erneuerbarer Energien von über 40 Prozent vor. Hier seien vor allem Windkraft, Photovoltaik und konzentrierte Solarenergie vorgesehen. Außerdem sei Energiearmut ein großes Thema in Südafrika, da 30 Prozent der Südafrikaner keinen Zugang zu Elektrizität hätten.

In der internationalen Politik trete Südafrika aktiv für nachhaltige Entwicklung ein. So habe Südafrika den Weltgipfel der Vereinten Nationen für Nachhaltige Entwicklung in Johannesburg 2002 und die Klimaverhandlungen in Durban 2011 ausgerichtet. Im Mai 2012 habe zudem das Stakeholder-Forum der EU-Afrika Energiepartnerschaft in Kapstadt stattgefunden. Themen waren unter anderem Investitionen und politische Rahmenbedingungen zur Förderung erneuerbarer Energien. Nach Angaben von Smith-Höhn ist Südafrika in der internationalen Politik vor allem an Technologietransfers, Kapazitätsaufbau und ,best practices' interessiert. Zudem betone die südafrikanische Regierung, mit erneuerbaren Energien auch die inländische Wertschöpfung und die Schaffung von Arbeitsplätzen fördern zu wollen. Klauseln über inländische Wertschöpfungen (Local-Content-Klauseln) seien in Südafrika daher von zentraler Bedeutung.

\section{Internationale Kooperationsansätze}

Die folgenden Vorträge befassten sich mit der internationalen Kooperation zur Förderung erneuerbarer Energien. Im Fokus stand mit der IRENA die bisher einzige internationale Organisation, die sich auf die Förderung erneuerbarer Energien konzentriert. Daneben wurde der weitere institutionelle Rahmen zur internationalen Förderung erneuerbarer Energien thematisiert.
Francisco Boshell präsentierte zunächst zentrale Rahmendaten und die inhaltliche Ausrichtung der IRENA. Sie verfolge das Ziel, die nachhaltige Nutzung erneuerbarer Energien weltweit voranzutreiben. Dies umfasse Biomasse, Erdwärme, Wasserkraft, Meeresenergie, Solar- und Windenergie. Der Hauptsitz sei in Abu Dhabi, in Bonn befände sich das Innovations- und Technologiezentrum der IRENA. Die IRENA habe bereits heute eine globale Reichweite: 100 Staaten seien der IRENA beigetreten, weitere 58 Staaten hätten die Statuten unterzeichnet und müssten diese noch ratifizieren. Mittelfristig verfolge die IRENA die Vision, sich als zentrale Plattform für internationale Kooperation und als Exzellenz- und Wissenszentrum zu etablieren, um Staaten bei der Transition hin zu erneuerbaren Energien zu unterstützen. Boshell stellte klar, dass die IRENA weder eine Bank noch eine Implementierungsagentur sei. Die IRENA möchte vielmehr als globale Stimme für erneuerbare Energien, als Beratungsquelle für Staaten und als Knotenpunkt internationaler Zusammenarbeit dienen. Dabei konzentriere sie sich auf drei Arbeitsbereiche: Innovation und Technologie, Wissensmanagement und Technologiekooperation sowie Politikberatung und Kapazitätsaufbau.

Anschließend präsentierte Boshell einige Errungenschaften der noch jungen Organisation: Die IRENA habe sich sehr früh auf die Entwicklung programmatischer Aktivitäten konzentriert, die sie gemeinsam mit verschiedenen Stakeholdern durchführe. Sie werde bereits als globale Plattform anerkannt und sei ein vielgesuchter Kooperationspartner. Zudem habe sie bereits verschiedene politikorientierte und technische Publikationen veröffentlicht und Mitgliedstaaten auf Anfrage beraten. Daneben nehme sie an zentralen globalen Initiativen teil, wie zum Beispiel an der Initiative der Vereinten Nationen ,Nachhaltige Energie für alle'. Die IRENA werde nun ihre Vision weiter umsetzen und dabei anstreben, einen minimalen Verwaltungsaufwand mit maximaler Wirkung zu verbinden. Sie stünde vor der Herausforderung, ihre mehr als 150 Mitgliedstaaten sinn- 
voll in ihre Aktivitäten einzubinden und eine Balance zwischen guter Politikberatung und dem Eintreten für erneuerbare Energien zu finden.

Sybille Röhrkasten stellte heraus, dass die Förderung erneuerbarer Energien ein relativ junger, jedoch sehr dynamischer Bereich internationaler Zusammenarbeit sei. Die Förderung erneuerbarer Energien adressiere drei zentrale globale Herausforderungen: Energieknappheit, Klimawandel und weitere Umweltrisiken sowie Energiearmut. Analog zu dieser dreifachen Herausforderung betreffe sie drei Bereiche internationaler Kooperation: Handels-, Klima- und Entwicklungspolitik.

Verschiedene Organisationen und Institutionen seien für die weltweite Förderung erneuerbarer Energien relevant. Neben der IRENA richteten zum Beispiel die MultistakeholderInitiativen ,Renewable Energy Policy Network for the 21st Century' (REN21), REEEP und GBEP einen exklusiven Fokus auf erneuerbare Energien. Die Internationale Energieagentur (IEA) decke neben erneuerbaren Energien auch fossile Energieträger ab. Die Weltbank, Regionale Entwicklungsbanken und verschiedene Organisationen der Vereinten Nationen förderten erneuerbare Energien in ihrer Entwicklungskooperation. Auch das Rahmenübereinkommen der Vereinten Nationen für Klimawandel ${ }^{1}$ biete wichtige Anstöße. In der Welthandelsorganisation (WTO) seien erneuerbare Energien noch kein großes Thema, obwohl sich Handelspolitiken entscheidend auf die weltweite Verbreitung erneuerbarer Energien auswirkten. Zudem seien die Foren der ,G8' und ,G20` aufgrund ihrer Macht, die internationale Agenda zu beeinflussen, und ihres politikfeldübergreifenden Ansatzes besonders geeignet, für die Förderung erneuerbarer Energien einzutreten.

Innerhalb dieses institutionellen Kontextes könne die IRENA eine entscheidende Rolle spielen. Um sich als globale Stimme für erneu- erbare Energien zu etablieren, seien einige Schritte notwendig. Die IRENA solle bestehende Erfahrungen und Wissen internationaler Kooperationen zusammenführen und auswerten, internationales Handeln koordinieren und lenken sowie in anderen Kooperationsbereichen Bewusstsein für erneuerbare Energien schaffen und die Agenda entsprechend beeinflussen. Dafür solle sie ihre Aktivitäten stärker als bisher auf internationale Organisationen und Prozesse ausrichten. Mit der IEA, REN21, der Weltbank und relevanten Organisationen der Vereinten Nationen könne sie eine umfassende Wissensdatenbank aufbauen. Im Bereich der Klima- und Entwicklungspolitik könne sie zunächst Transparenz über bisherige Aktivitäten zur Förderung erneuerbarer Energien herstellen und darüber hinaus, best and worst practices' auswerten und kommunizieren. Im Bereich der Handelspolitik solle sie Querverbindungen zur Förderung erneuerbarer Energien und damit verbundene Chancen und Risiken analysieren. Des Weiteren solle die IRENA die Agenda von G8 und G20 beeinflussen und beiden Foren ihre Beratungstätigkeiten anbieten.

Gerhard Knies sprach sich für die Bildung einer institutionellen Struktur aus, die weit über bisherige Kooperationsansätze zur Förderung erneuerbarer Energien hinausgeht. Das zentrale Argument für erneuerbare Energien sei der Klimaschutz. Das Zwei-Grad-Ziel erfordere eine vollständige Beendigung der Nutzung fossiler Energien, lange bevor die bekannten Reserven aufgebraucht seien. Für die Abschaffung fossiler Energien sei ein organisierter und kontrollierter Prozess erforderlich. Ausreichend finanzielle, technische und natürliche Ressourcen seien vorhanden. Knies schlug daher die Gründung einer internationalen Agentur zur Beseitigung fossiler Energien vor. Diese solle Szenarien für einen Ausstieg aus fossilen Energien entwickeln und darüber entscheiden, welche der vorhandenen Reserven noch verbraucht werden dürften. Zudem seien saubere Energiequellen zu identifizieren und

1 United Nations Framework Convention on Climate Change (UNFCCC). 
ökonomische Alternativen für diejenigen zu entwickeln, die ihre fossilen Energiereserven nicht mehr nutzen dürften. Ein globaler Rahmen müsse die illegale Nutzung fossiler Energiereserven verhindern.

\section{Die Einbindung der Schwellenländer}

Die beiden letzten Vorträge behandelten Ansätze zur Einbindung von Schwellenländern in die internationale Governance. Hierbei wurden zunächst die EU-Energiedialoge mit Schwellenländern vorgestellt. Der abschließende Vortrag weitete den Blick auf die internationale Governance im Allgemeinen und analysierte sowohl Konflikte und Herausforderungen als auch vielversprechende Kooperationsinitiativen.

Marion Wilde stellte zunächst die politischen Prioritäten der Europäischen Union in ihren Energie-Außenbeziehungen vor. Neben der Weiterentwicklung der externen Dimension des Binnenmarktes strebe die Europäische Union Partnerschaften für eine sichere, nachhaltige und wettbewerbsfähige Energieversorgung an. Sie setze sich dabei auch für den $\mathrm{Zu}-$ gang zu nachhaltiger Energie in Entwicklungsländern ein. Des Weiteren engagiere sie sich für eine internationale Verbreitung europäischer Politikansätze.

Die Energie-Kooperation mit China sei bisher am umfassendsten. Schwerpunkte des EUChina Energiedialogs seien saubere Kohletechnologien, erneuerbare Energien und intelligente Stromnetze (, smart grids ${ }^{\circ}$ ) sowie Energiesicherheit. Im Mai 2012 habe ein erstes EUChina High Level Meeting on Energy stattgefunden, an dem neben dem Kommissionspräsidenten José Manuel Barroso und dem chinesischen Vize-Premier Li Keqiang Repräsentanten aller 27 EU-Mitgliedstaaten teilgenommen hätten. Neben einer gemeinsamen Erklärung über Energiesicherheit sei eine verstärkte Kooperation im Elektrizitätsmarkt vereinbart worden. Seit April 2010 existiere das ,EU-China Clean Energy Centre', welches in China saubere Kohletechnologien, Biokraftstoffe, er- neuerbare Energien und Energieeffizienz fördere. Das europäisch-chinesische Institut für saubere und erneuerbare Energie biete ein Masterprogramm für chinesische Studierende und eine interaktive Plattform für Energieexperten aus China und der Europäischen Union an. Weitere Projekte unterstützten chinesische Städte im Bereich Energie- und Ressourceneffizienz und förderten nachhaltige Produktion und Konsum in Asien.

Auch mit Indien, Brasilien und Südafrika kooperiere die Europäische Union im Energiebereich. Im Rahmen des 2005 etablierten EUEnergie-Panels befassten sich drei europäischindische Arbeitsgruppen mit sauberen Kohletechnologien, Kohle, erneuerbaren Energien und Energieeffizienz. Vertieft wurde die Kooperation auf dem EU-Indien-Gipfel im Februar 2012, wo eine gemeinsame Erklärung über verstärkte Energiekooperation verabschiedet wurde. Auf dem EU-Brasilien-Gipfel sei ein gemeinsames Komitee eingerichtet und ein gemeinsamer Aktionsplan verabschiedet worden. Der Sektor-Dialog zu Energie umfasse unter anderem Themen wie Energiesicherheit, Binnenmarkt und Nachhaltigkeit sowie erneuerbare Energien. Zudem befassten sich die Europäische Union und Brasilien im Rahmen des ,International Biofuels Forum‘ (IBF) mit nachhaltigen Biokraftstoffen und im Kontext der ,International Partnership for Energy Efficiency Cooperation' (IPEEC) mit den Themen kohlenstoffarme Energietechnologien und Energieeffizienz. Mit Südafrika kooperiere die Europäische Union seit dem EU-Südafrika-Gipfel 2008. Drei Arbeitsgruppen befassten sich mit $\mathrm{CO}_{2}$-Abscheidung und -Speicherung sowie mit sauberen Kohletechnologien, Energieeffizienz und Nukleartechnologie. Ein Gemeinsamer Kooperationsrat tage jährlich.

Thijs Van de Graaf unterstrich zunächst die Bedeutung einer Kooperation mit Schwellenländern für die Förderung erneuerbarer Energie. Schwellenländer gehörten zu den größten Produzenten von erneuerbaren Energien und böten das Potenzial, die am schnellsten wach- 
senden Märkte zu werden. Aufgrund des Stillstandes in den internationalen Klimaverhandlungen sei die Verbreitung von erneuerbaren Energien in Schwellenländern zentral, um internationalen Klimaschutz voranzubringen. Hier ergebe sich ein besonderes Potenzial, da die großen Emittenten von Treibhausgasen gleichzeitig wichtige Akteure im Handel mit erneuerbaren Energien seien. Internationale Kooperation sei zentral, um die weltweite Verbreitung erneuerbarer Energien zu beschleunigen. Sie müsse vor allem faire Rahmenbedingungen im Vergleich zu fossilen Energieträgern schaffen und schwerwiegende Handelskonflikte verhindern. So erhebe die Europäische Union höhere Handelsschranken auf Ethanol als auf Erdöl. Zudem werde das wirtschaftliche Wetteifern um saubere Energie fälschlicherweise oft als Nullsummenspiel gesehen und nicht als etwas, von dem alle profitieren können.

Van de Graaf identifizierte drei zentrale Konfliktlinien und damit verbundene Herausforderungen für internationale Kooperation: Geopolitik, Widersprüche ,grünen Wachstums“ und divergierende nationale Entwicklungen. Der geopolitische Fokus gehe mit Protektionismus und Handelsbarrieren einher. Inhärente Widersprüche des Konzeptes eines ,grünen Wachstums' führten dazu, dass das Konzept zwar von allen gemocht, jedoch von niemandem implementiert werde. Unterschiedliche geografische Bedingungen gingen mit sehr unterschiedlichen Zusammensetzungen nationaler Energieversorgung einher. Daher sei die Bildung eines kohärenten globalen Rahmens schwierig. Gleichzeitig müsse sichergestellt werden, dass genügend Raum für nationale Entscheidungen bliebe.

Einige vielversprechende internationale Initiativen gäbe es bereits. So plane China die Einführung eines Emissionshandelssystems. Das ,U.S.-China Clean Energy Research Center ${ }^{6}$ zeige, dass China und die USA in der Energiepolitik nicht nur konkurrierten, sondern auch kooperierten. Auch die IRENA sei eine viel- versprechende Initiative. Das CEM böte den Vorteil, dass es auch private Akteure einbinde. Sein Erfolg zeige sich daran, dass andere Akteure CEM-Initiativen aufgriffen. Auch die Initiative der Vereinten Nationen ,Nachhaltige Energie für alle' und der Rio+20-Prozess stellten wichtige Schritte dar. Insbesondere die Verhandlungen $\mathrm{zu}$ nachhaltigen Entwicklungszielen könnten entscheidende Impulse für erneuerbare Energien liefern. Van de Graaf stellte abschließend die Handelspolitik als einen zentralen Bereich für die Förderung erneuerbarer Energien heraus. Ein Handelsabkommen über nachhaltige Energie sei dringend notwendig, um faire Rahmenbedingungen für erneuerbare Energien zu schaffen.

Insgesamt zeigte die Konferenz, dass sich sowohl in der internationalen Politik als auch innerhalb der betrachteten Schwellenländer China, Indien, Brasilien und Südafrika zunehmend die Erkenntnis durchsetzt, dass ein umfassender Ausbau erneuerbarer Energien unabdingbar ist. Wichtige politische Initiativen wurden bereits ergriffen. Für eine umfassende Energietransition reichen diese jedoch bei Weitem nicht aus. Die energiepolitischen Rahmenbedingungen einzelner Staaten variieren stark, je nach geografischen Bedingungen und institutionellen Faktoren. Daraus resultieren auch sehr unterschiedliche Interessen und Schwerpunkte in der internationalen Politik. Internationale Kooperation kann vor allem mit Wissens- und Erfahrungsaustausch zur Förderung erneuerbarer Energien beitragen. Der noch jungen internationalen Organisation IRENA kommt hier eine besondere Bedeutung zu. Wie in anderen Politikbereichen auch, muss in der internationalen Energiepolitik ein Umdenken erfolgen. Mit den Schwellenländern gibt es nun eine neue Gruppe international einflussreicher Staaten, die das weitverbreitete Denken in Nord-Süd-Kategorien aufbrechen lässt. Das Blicken über den eigenen Tellerrand und das Hinterfragen eigener Konzepte wird daher immer wichtiger - nicht nur in der Politik, sondern auch in der Wissenschaft. 